\title{
Neurofilament light is a biomarker of brain involvement in lupus and primary Sjögren's syndrome
}

\author{
Anne B. Tjensvoll ${ }^{1}$ - Maria B. Lauvsnes ${ }^{2} \cdot$ Henrik Zetterberg $^{3,4,5,6} \cdot$ Jan T. Kvaløy $^{7,8} \cdot$ Ingeborg Kvivik $^{7}$. \\ Stian S. Maroni ${ }^{9}$. Ole J. Greve ${ }^{10}$. Mona K. Beyer ${ }^{11,12}$. Shunsei Hirohata ${ }^{13}$. Chaim Putterman ${ }^{14,15,16}$. Guido Alves ${ }^{17,18}$. \\ Erna Harboe $^{2} \cdot$ Kaj Blennow $^{3,4} \cdot$ Lasse G. Gøransson ${ }^{2,19} \cdot$ Roald Omdal $^{2,19}$
}

Received: 9 July 2020 / Revised: 20 October 2020 / Accepted: 20 October 2020 / Published online: 30 October 2020

(c) The Author(s) 2020

\begin{abstract}
Background To test the hypothesis that neurofilament light (NfL) in CSF is a biomarker of CNS involvement in patients with systemic lupus erythematosus (SLE) and primary Sjögren's syndrome (pSS), we measured NfL in CSF from 52 patients with lupus and 54 with pSS and explored associations with clinical, structural, immunological and biochemical abnormalities.

Methods In CSF, we measured NfL, anti-P antibodies, protein S100B and TWEAK by ELISA and anti-NR2 antibodies by electrochemiluminescence. Anti-phospholipid antibodies and routine immunological tests were performed in blood. IgG and albumin were measured in CSF and serum for assessment of the blood-brain barrier function (Q-albumin) and intrathecal IgG production (IgG index). Cerebral MRI and neuropsychological testing were performed.

Results A multivariable regression model showed that increasing CSF anti-NR2 antibody levels were associated with increasing NfL levels in patients with SLE $(B 1.27,95 \%$ CI $0.88-1.65, p<0.001)$. Age contributed significantly in the model $(B$ $0.04,95 \%$ CI $0.03-0.05, p<0.001)$. Similar findings were observed in the pSS group. Adjusted for age and sex, no associations were found between NfL levels and any MRI data. In SLE patients, higher NfL concentrations were associated with impairments in psychomotor speed and motor function, and in pSS with motor dysfunction. These associations remained in multivariable regression models.

Conclusions Increased concentration of NfL in CSF is a marker of cerebral involvement in patients with SLE and pSS, is strongly associated with the presence of anti-NR2 antibodies, and correlates with cognitive impairment in several domains.
\end{abstract}

Keywords Neurofilament light chain · Anti-NR2 antibodies · Cognitive dysfunction · Systemic lupus erythematosus · Primary Sjögrens's syndrome

\section{Introduction}

Systemic lupus erythematosus (SLE) is a chronic systemic inflammatory autoimmune disease that frequently involves the CNS [1]. The manifestations are diverse, varying from life-threatening strokes and encephalitis to headaches and mood disorders. Primary Sjögren's syndrome (pSS) is another systemic inflammatory autoimmune disease that primarily attacks exocrine glands, such as the salivary and

Electronic supplementary material Supplementary information is available for this paper at https://doi.org/10.1007/s00415-02010290-y.

Roald Omdal

roald.omdal@lyse.net

Extended author information available on the last page of the article lacrimal glands, leading to dryness of the mouth and eyes [2]. Neuropsychiatric phenomena are common in both diseases [3].

Neurofilament light chain protein $(\mathrm{NfL})$ is one out of five neurofilament subunits that comprise the neuronal cytoskeleton. Increased NfL levels in CSF reflect axonal damage and degeneration; consequently, $\mathrm{NfL}$ is frequently used as a marker of CNS injury in neurodegenerative conditions, multiple sclerosis, cerebrovascular diseases, and traumatic brain injury (Fig. 1) [4]. We hypothesized that NfL levels in CSF would be increased in patients with SLE and/or pSS. We also hypothesized that increased NfL levels might be associated with brain reactive antibodies, such as anti-phospholipid antibodies (aPL antibodies) [5, 6], antibodies against the NR2 subunit of the $N$-methyl-D-aspartate receptor (anti-NR2 


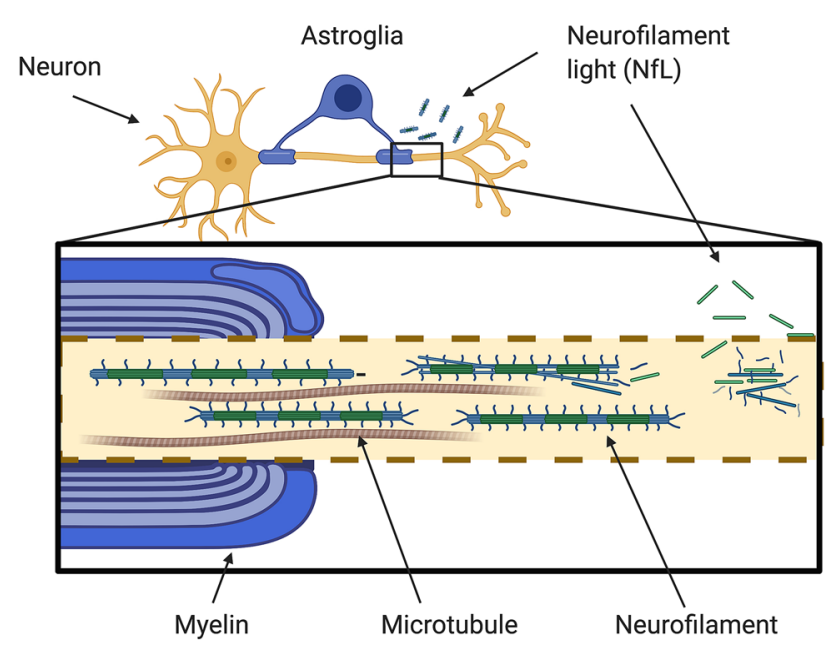

Fig. 1 Neuron with neurofilaments. Figure illustrates disintegrating neurofilaments, and neurofilament light chains (NfL) leaking out of the axon. Figure created with BioRender.com

antibodies) [7, 8], or antibodies directed against ribosomal P proteins (anti-P antibodies) [9].

The present study aimed to determine whether CSF NfL could serve as a biomarker of CNS involvement in SLE and/ or pSS. To that end, we investigated potential associations between NfL concentrations and structural, immunological, and biochemical abnormalities in patients with SLE and pSS. In addition, we investigated whether functional abnormalities were evident in patients with increased NfL levels, exemplified by cognitive dysfunction or headache.

\section{Methods}

Nearly, all patients with systemic autoimmune diseases in Rogaland County, Norway, are allocated to Stavanger University Hospital, where this study was performed. Recruitment was based on hospital records from in- and outpatients.

\section{SLE group}

Eighty-six patients, all Caucasian, fulfilled the 1982 revised American College of Rheumatology (ACR) criteria for SLE [10]. Of these, 70 (81\%) consented to participate in the study. Two patients withdrew consent, and one was excluded, due to a brain tumor. Thus, 67 patients (78\%) were included in the study.

\section{PSS group}

Seventy-two (73\%) out of 99 patients, all Caucasian, fulfilled the American European Consensus Group (AECG) criteria for pSS [11], and consented to participate in the study. One patient was excluded due to a brain tumor. Thus, $71(72 \%)$ patients were included.

\section{Clinical examination}

All patients were examined by two internists (EH and LG) and a neurologist (ABT) during a 2-day stay in the hospital, for research purposes only. SLE disease activity was assessed with the SLE disease activity index (SLEDAI) [12], and organ damage was assessed with The Systemic Lupus International Collaborating Clinics/American College of Rheumatology Damage Index (SLICC/ACR-DI) [13]. Headache was assessed in a structured interview, and classified according to the International Classification of Headache Disorders (ICHD II) [14]. Depression was assessed with the Beck Depression Inventory (BDI) applying a cut-off score of $\geq 13$ to identify current clinical depression [15]. Fatigue severity was scored by the fatigue Visual Analogue Scale (fVAS) [16]. Arterial hypertension was defined as systolic blood pressure $\geq 140$ and/or diastolic pressure $\geq 90 \mathrm{mmHg}$ or current use of antihypertensive medication.

\section{Lumbar puncture}

Fifty-two of the 67 patients with SLE (78\%) and 54 of the 71 patients with pSS (76\%) underwent lumbar punctures. All CSF samples were obtained between 1 and 2 p.m., placed on ice, and centrifuged at $4{ }^{\circ} \mathrm{C}$ at $3000 \times g$ for $10 \mathrm{~min}$. Supernatants were immediately aliquoted and frozen at $-70{ }^{\circ} \mathrm{C}$ until analysis.

\section{Laboratory analyses}

\section{CSF}

IgG was measured in CSF and serum with the Cobas Integra Immunoglobulin $\mathrm{G}$ (Turbidimetric) assay, and albumin with Tina-quant a Albumin Gen.2 (Roche Diagnostics, Mannheim, Germany) according to the manufacturer's instructions. The CSF/serum albumin ratio (Q-albumin) was calculated as [CSF-albumin/serum-albumin] as a measure of the blood-brain barrier function, while the $\mathrm{IgG}$ index was calculated as [(CSF-IgG/serum-IgG)/(CSF-albumin/ serum-albumin)] as a measure for intrathecal IgG production, and chronic CNS inflammation [17]. Anti-NR2 antibodies were detected by electrochemiluminescence [8] and anti-P antibodies by ELISA [18], both as previously described. Protein S100B was analyzed with the Human S100B ELISA kit (Abnova, Jhongli City, Taiwan) according to the manufacturer's instructions, and TNF-like weak inducer of apoptosis (TWEAK) by ELISA (R\&D Systems, Minneapolis, MN, USA) as previously described [19]. CSF $\mathrm{NfL}$ concentration was measured using a commercially 
available ELISA according to instructions from the manufacturer (UmanDiagnostics, Umeå, Sweden). The measurements were performed in one round of experiments by board-certified laboratory technicians who were blinded to the clinical data. Intra-assay coefficients of variation were below $10 \%$.

\section{Blood}

Routine biochemical, hematological, and immunological analyses were performed at the hospital's laboratories. ANA was detected with the HEp-2000 assay (Immunoconcepts, Sacramento, CA, USA), and presence of anti-doublestranded (ds) DNA by Nova Lite dsDNA Crithidia luciliae 708,200 indirect immunofluorescence assay (Nova Diagnostics, San Diego, CA, USA). Anti-SSA, and anti-SSB antibodies were measured by ELISA with QUANTA Lite ENA 6 assay (Inova Diagnostics, San Diego, CA, USA), and positive results confirmed by Quanta Lite SSA and SSB ELISA (Inova Diagnostics). Anti-cardiolipin IgM and IgG antibodies were measured with the QUANTA Lite ${ }^{\mathrm{TM}}$ ACA IgM and IgG ELISA (Inova Diagnostics). Lupus anticoagulant was measured by the activated partial thromboplastin time and dilute Russell's viper venom time (Dade Behring, Marburg, Germany). Anti-phospholipid (aPL) antibodies were considered present if the patient had a positive anti-cardiolipin IgM- or IgG-antibody test, was lupus-anticoagulant positive, or any combinations of these.

\section{MRI}

MRI examinations were performed with a 1.5-T Philips Gyroscan NT Intera Release 10 (Philips Medical Systems, Best, The Netherlands). White matter hyperintensities (WMHs) were assessed in accordance with the semi-quantitative visual rating scale of Scheltens et al. [20]. Global GM and WM volumes were estimated using the VBM8 extension of the SPM8 software. Details of the MRI protocols and preprocessing are previously described [21].

\section{Neuropsychological testing}

The tests were administered by a trained psychometric test technician. Results were analyzed by a clinical neuropsychologist (SSM). The test batteries included the Wechsler Memory Scale, Revised (WMS-R) [22], Wechsler Adult Intelligence Scale (WAIS) [23], Stroop Color-Word Interference Test [24], Wisconsin Card Sorting Test [25], FAS Verbal Influency Test [26], Tactual Performance Test (TPT), Fingertapping test, Trail Making Test A and B, Category Test, Seashore Rhythm Test, Lafayette's Hand Dynamometer Test, and the Lafayette Grooved Pegboard Test [27]. These tests mapped functions in eight cognitive domains: memory, psychomotor speed, visual-spatial processing, motor function, language, reasoning/problem solving, simple attention and complex attention. Scores were compared to normative data for each test, and cut-off score for abnormality defined by a standardized score $\geq 2$ SD from the reference mean. Cognitive dysfunction was defined as abnormality in one or more of these domains. The neuropsychological tests are based on normative data that are adjusted for age, sex and education.

\section{Statistics}

Continuous data are reported as medians and ranges. Categorical data are reported as numbers and percentages. Differences between groups were evaluated with the chi-square test for categorical data, and the Mann-Whitney $U$ test for continuous data. NfL measurements were log-transformed to achieve a less skewed distribution, more appropriate for use in the statistical analyses. Linear regression analyses were performed to examine potential explanatory variables for $\mathrm{NfL}$ in CSF. All regression analyses with $\mathrm{NfL}$ as response variable were corrected for age by including age as adjustment variable. In the analyses of demographic and clinical variables, the explanatory variables were disease duration, hypertension, education, SLICC-DI and SLEDAI.

In the analyses with laboratory data, explanatory variables were sex, anti-NR2 in blood, aPL antibodies in blood and the following variables measured in CSF: anti-NR2 antibodies, anti-P antibodies, TWEAK, protein S100B and IgG. We first tested one explanatory variable at a time, adjusted for age. Then, we ran backward model selection, and the final model is reported. MRI analyses were performed with WMHs, global GM and-WM as response variables, $\mathrm{NfL}$ as explanatory variable and sex and age as adjustment variables.

Logistic regression analyses were performed to examine whether NfL was associated with abnormal cognitive domain scores. We ran both univariable analyses with only $\operatorname{logNfL}$ as explanatory variable, and each of the domain scores (dichotomized into normal/abnormal) as response, and multivariable analyses with $\operatorname{logNfL}$, anti-NR2 antibodies, anti-P antibodies, TWEAK, and protein S100B as explanatory variables. Similar analyses were performed with clinical variables such as headaches, depression, or fatigue as response variables. Correction for multiple testing was not performed. $P$ values $<0.05$ were considered significant.

\section{Results}

Selected clinical, laboratory and imaging data are shown in Table 1. SLE patients were younger than $\mathrm{pSS}$ patients. There were more cerebral infarcts in the SLE group, probably 
Table 1 Selected demographic and clinical data in SLE- and pSS patients

\begin{tabular}{llll}
\hline & SLE $(n=67)$ & pSS $(n=71)$ & $p$ Value \\
\hline Women/men, $n(\%)$ & $58 / 9(87 / 13)$ & $61 / 10(86 / 14)$ & $>0.99$ \\
Age, years & $42.4(20-76)$ & $58.1(27-87)$ & $<0.001$ \\
Education, years & $13(7-20)$ & $12(7-20)$ & 0.34 \\
Disease duration, years & $11.0(1-32)$ & $6.1(0-24)$ & $<0.001$ \\
SLEDAI scores & $2.0(0-26)$ & NA & NA \\
ANA positive, $n(\%)$ & $65(97)$ & $59(83)$ & 0.007 \\
Anti-SSA/SSB positive, $n$ & $22(33)$ & $56(79)$ & $<0.001$ \\
$\quad(\%)$ & & & \\
aPL positive, $n(\%)$ & $26(39)$ & $9(13)$ & 0.001 \\
BDI score & $6.0(0-27)$ & $9.0(0-38)$ & 0.03 \\
Fatigue VAS score & $49(1-98)$ & $65(3-96)$ & 0.07 \\
Arterial hypertension, $n(\%)$ & $34(51)$ & $43(61)$ & 0.25 \\
Current medications & & & \\
$\quad$ Corticosteroids, $n(\%)$ & $44(66)$ & $16(23)$ & $<0.001$ \\
$\quad$ Antimalarials, $n(\%)$ & $33(49)$ & $26(37)$ & 0.13 \\
MRI findings & & & \\
$\quad$ Cortical infarcts, $n(\%)$ & $7 / 62(11)$ & $0 / 68$ & 0.005 \\
Lacunar infarcts, $n(\%)$ & $8 / 62(13)$ & $2 / 68(3)$ & 0.05 \\
\hline
\end{tabular}

Continuous data reported as median and ranges. Categorical data reported as numbers and percentages. The Mann-Whitney test was used to test for differences between the groups for continuous variables and the chi-square test for categorical variables

$S L E$ systemic lupus erythematosus, $p S S$ primary Sjögren`s syndrome, SLEDAI SLE disease activity index, ANA antinuclear antibodies, SSA Sjögren`s syndrome A antigen, $S S B$ Sjögren`s syndrome B antigen, $a P L$ anti-phospholipid antibodies, BDI Beck Depression Inventory, $V A S$ visual analog scale

reflecting the higher prevalence of aPL antibodies compared with pSS patients. The pSS patients had lower mood than patients with SLE.

\section{NfL in CSF and demographic and clinical variables}

CSF NfL levels were higher in pSS than SLE patients (Table 2), but after adjusting for age by a linear regression analysis, no significant difference in NfL between groups remained. The $\mathrm{NfL}$ levels increased with increasing age in both SLE $(B 0.003,95 \%$ CI $0.002-0.005, p<0.001)$ and pSS patients ( $B$ 0.002, 95\% CI 0.001-0.003, $p=0.007)$ (Fig. 2). Sex, disease duration or education did not influence NfL levels, neither in SLE patients nor in pSS patients. No associations were found between NfL levels and SLEDAI or SLICC-DI scores in the SLE patients (data not shown).

NfL levels were not associated with hypertension, fVAS scores, BDI scores, headaches in general or migraines in particular in either patient group (data not shown). In pSS patients, tension type headache was associated with higher NfL concentrations (OR 0.25, $p=0.02$ ).

\section{NfL and other laboratory data}

Measures of anti-NR2- and anti-P antibodies, protein S100B, TWEAK and IgG index in CSF are shown in Table 2. S100B and TWEAK were higher in pSS patients compared with SLE, but adjusted for age, only the difference for TWEAK remained ( $B$ 453.2, 95\% CI 80.5-825.9, $p=0.02$ ).

In regression analyses, with $\mathrm{NfL}$ as response variable, we found that $\mathrm{NfL}$ levels increased with increasing CSF concentrations of anti-NR2 antibodies in both SLE $(B 1.26$, 95\% CI $0.83-1.69, p<0.001)$ and pSS patients $(B 0.54$, $95 \%$ CI $0.24-0.84, p=0.001$ ) (Fig. 3). No associations were revealed between anti-NR2 antibodies in blood and NfL in CSF in SLE $(B-0.11,95 \% \mathrm{CI}-0.45-0.23, p=0.53)$ or pSS patients $(B 0.09,95 \% \mathrm{CI}-0.16-0.34, p=0.48)$.

Increasing levels of $\mathrm{NfL}$ were associated with increasing CSF concentrations of anti-P antibodies in pSS patients $(B$ 27.0, 95\% CI 10.6-43.3, $p=0.002)$, but not in SLE ( $B$ 11.6, $95 \%$ CI $-2.4-25.7, p=0.10$ ).

Furthermore, no association was revealed between NfL and S100B in SLE patients $(B$ 0.001, 95\% CI - 0.003-0.006, $p=0.59)$, or in the pSS patients $(B 0.001,95 \%$ CI $-0.001-0.003, p=0.56$ ).

A positive association was estimated between NfL and TWEAK in pSS patients, although not statistically

Table 2 CSF analyses

\begin{tabular}{lllr}
\hline & SLE $(n=67)$ & pSS $(n=71)$ & $p$ Value \\
\hline NfL pg/mL & $492(133-18,608)(n=47)$ & $764(214-10,439)(n=49)$ & 0.008 \\
Anti-NR2 ab & $0.38(0.1-2.2)(n=52)$ & $0.41(0.2-3.0)(n=54)$ & 0.366 \\
Anti-P ab ug/mL & $<0.001(<0.001-0.13)(n=51)$ & $<0.001(<0.001-0.04)(n=54)$ & 0.119 \\
S100B pg/mL & $222(109.5-419.8)(n=50)$ & $264(137.8-544.3)(n=54)$ & 0.003 \\
TWEAK pg/mL & $887(369-2351)(n=50)$ & $1351(481-5184)(n=52)$ & $<0.001$ \\
IgG index & $0.53(0.45-1.57)(n=52)$ & $0.52(0.41-2.05)(n=54)$ & 0.175 \\
\hline
\end{tabular}

Data reported as median and ranges. P values calculated by the Mann-Whitney test

$S L E$ systemic lupus erythematosus, $p S S$ primary Sjögren`s syndrome, $N f L$ neurofilament light, $a b$ antibody ${ }^{a}$ value given as a ratio of signal against an internal calibrator with defined signal intensity; TWEAK, TNFlike weak inducer of apoptosis 


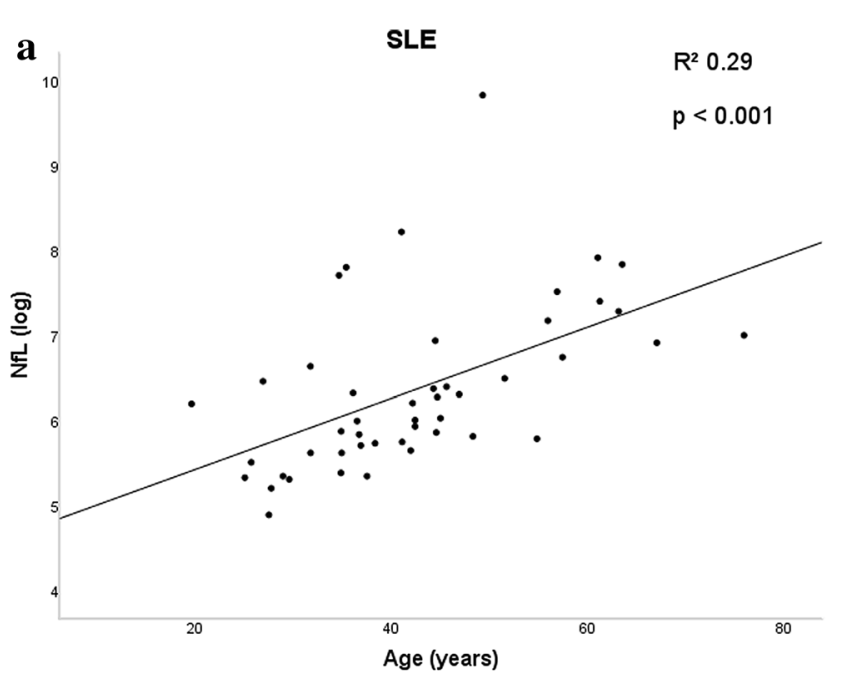

$\mathrm{NfL}(\log )=4.6+0.04 \times$ age $($ years $)$

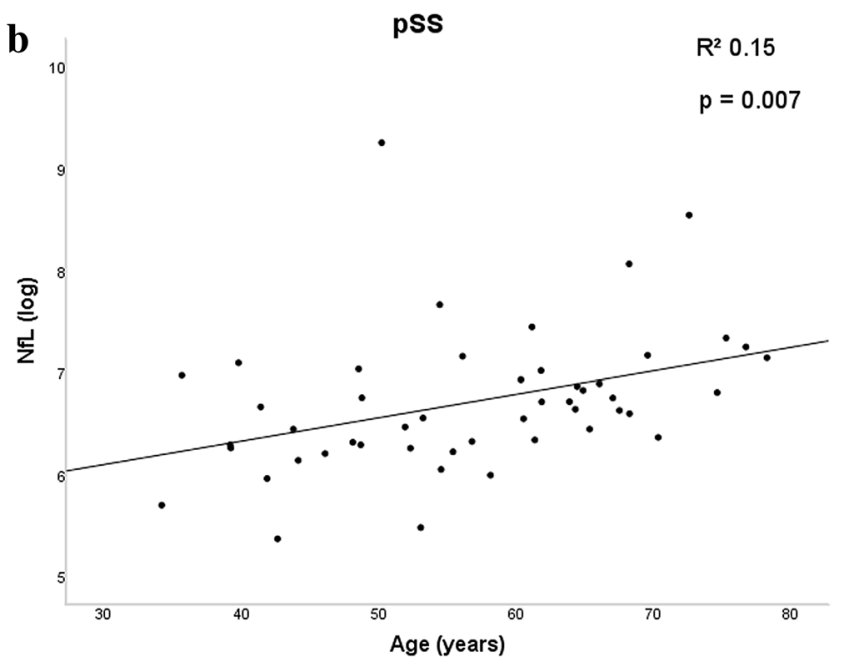

$\mathrm{NfL}(\mathrm{log})=5.4+0.02 \times$ age (years)

Fig. 2 Influence of age on CSF NfL concentration. Results are shown for patients with (a) systemic lupus erythematosus $(N=47)$ and $(\mathbf{b})$ primary Sjögren's syndrome $(N=49)$

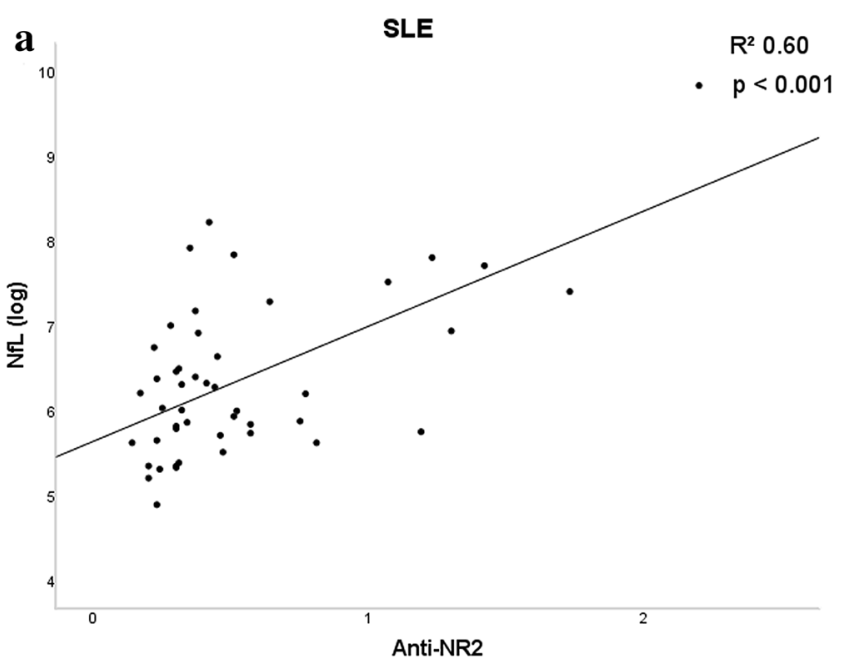

$\mathrm{NfL}(\log )=5.6+1.3 \times$ anti-NR2

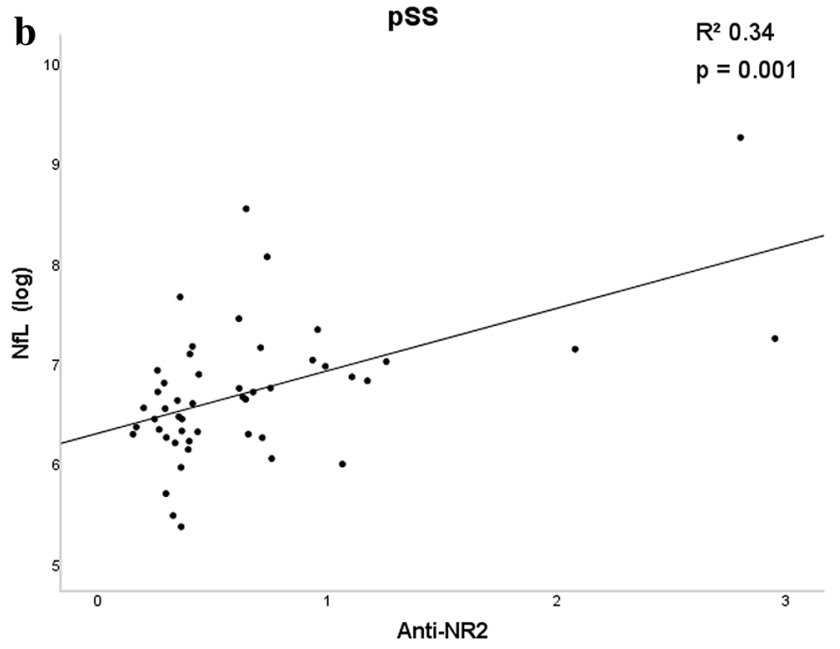

$\mathrm{NfL}(\log )=6.3+0.5 \times$ anti-NR2

Fig. 3 Associations between NfL and anti-NR2 antibodies in CSF. Results are shown for patients with (a) systemic lupus erythematosus $(N=47)$ and (b) primary Sjögren's syndrome $(N=49)$

significant $(B$ 0.00016, 95\% CI - 0.000009-0.00033, $p=0.06)$. In the SLE patients, the association was not significant either $(B \quad 0.00048,95 \%$ CI $-0.00016-0.0011$, $p=0.14$ ).

Intrathecal IgG increased with increasing NfL in SLE ( $B$ $0.02,95 \%$ CI $0.004-0.042, p=0.02$ ), but not in pSS patients (B $0.004,95 \%$ CI $-0.002-0.010, p=0.16$ ).

A positive association was estimated between Q-albumin and NfL levels in SLE, however, not statistically significant ( $B$ 0.10, 95\% CI $-0.005-0.214, p=0.06$ ), while not in pSS patients $(B 0.03,95 \% \mathrm{CI}-0.074-0.130, p=0.59)$.
No associations were revealed between NfL and ANA or IgG indices in the two patient groups (data not shown).

In a multivariable regression model with $\log \mathrm{NfL}$ as response variable, age as adjustment variable, and sex, aPL in blood, and anti-NR2-, anti-P antibodies, S100 $\mathrm{B}$, TWEAK and IgG in CSF as explanatory variables, increasing levels of anti-NR2 antibodies were associated with increasing levels of NfL in the SLE patients (B 1.27, 95\% CI 0.88-1.65, $p<0.001$ ). Age also contributed significantly in the model, while no contribution of sex, anti-P antibodies, S100B, IgG, TWEAK or aPL was 
found. Also in the pSS group, increasing NfL levels were associated with anti-NR2 antibodies in CSF ( $B$ 0.54, 95\% CI 0.24-0.84, $p=0.001)$. Final models are shown in Supplementary Table 1.

\section{NfL and MRI}

In these analyses, we used MRI data as response variable, and because both age and sex can influence cerebral volumes, these variables were included as adjustment variables in the regression analyses with $\mathrm{NfL}$ as explanatory variable. No associations were evident between $\mathrm{NfL}$ levels and WMHs, global GM-, WM-, or hippocampus volumes in SLE or pSS patients (data not shown).

\section{Neuropsychological tests}

Cognitive dysfunction defined as dysfunction in one or more domains, was evident in $30(45 \%)$ of the 67 SLE patients and 35 (49\%) of the pSS patients, $p=0.60$. No differences between SLE- and pSS patients were evident regarding cognitive dysfunction in the specific domains (data not shown).

\section{NfL and neuropsychological data}

In SLE patients, higher NfL concentrations were associated with impairment in psychomotor speed and motor function (Table 3 ). The associations remained in multivariable regression models.

In pSS patients, higher NfL levels were associated with impaired motor function in both univariable and multivariable regression models.

\section{Discussion}

The main finding in this study was that increasing concentrations of NfL in CSF was associated with increasing levels of anti-NR2 antibodies in CSF, and was a marker of cognitive dysfunction in patients with SLE as well as in pSS. We also investigated a panel of other biomarkers for brain involvement, and although several associations between NfL and these biomarkers were evident, antiNR2 antibodies was the dominating actor with a strong and consistent association to NfL levels evident both in univariate and multivariate models. These findings complement previous studies demonstrating the pathogenetic potential of anti-NR2 antibodies for cerebral dysfunction in SLE and pSS [21, 28], and indicate that increased NfL levels in CSF reflect neuronal damage or dysfunction. These observations extend current knowledge in neurodegenerative-, inflammatory-, cerebral vascular disease, and head traumas, and show that NfL also in the two systemic inflammatory autoimmune diseases, SLE and pSS, can be regarded as a general biomarker of harmful neuronal CNS processes [29].

Only one previous study has investigated NfL in patients with SLE [30]. The authors of that study reported higher NfL levels in CSF in patients compared with healthy subjects. In addition, the highest NfL concentrations were found in SLE patients with NP involvement, and levels were lower after treatment with cyclophosphamide.

SLE and pSS are distinct and different diseases, but share similarities, such as an autoimmune pathogenesis, systemic inflammation and CNS involvement. NfL lacks disease specificity, and can be detected in low concentrations in the CSF of healthy persons and increases with increasing age [4]. We found a strong association between increasing $\mathrm{NfL}$ concentrations and increasing age both in
Table 3 Impact of increasing NfL levels in CSF on risk for cognitive dysfunction

\begin{tabular}{|c|c|c|c|c|}
\hline & \multicolumn{2}{|l|}{ SLE } & \multicolumn{2}{|l|}{$\mathrm{pSS}$} \\
\hline & OR $(95 \% \mathrm{CI})$ & $p$ Value & OR $(95 \% \mathrm{CI})$ & $p$ Value \\
\hline Memory & $0.33(0.07-1.63)$ & 0.17 & $1.63(0.58-4.65)$ & 0.36 \\
\hline Psychomotor speed & $3.57(1.48-8.64)$ & 0.005 & $1.76(0.72-4.30)$ & 0.21 \\
\hline Visual-spatial processing & $1.35(0.38-4.79)$ & 0.65 & $3.75(0.83-16.95)$ & 0.09 \\
\hline Motor function & $2.28(1.09-4.76)$ & 0.03 & $9.07(1.84-44.84)$ & 0.007 \\
\hline Language & $1.06(0.47-2.40)$ & 0.89 & $0.94(0.24-3.70)$ & 0.92 \\
\hline Reasoning /problem solving & NA & & NA & \\
\hline Simple attention & $0.54(0.10-2.81)$ & 0.47 & NA & \\
\hline Complex attention (executive) & $1.8(0.90-3.61)$ & 0.10 & $0.68(0.26-1.79)$ & 0.43 \\
\hline
\end{tabular}

The table shows the increase in risk for cognitive dysfunction related to a one unit increase in log-NfL for each function score and patient group

$C I$ confidence interval, $O R$ odds ratio, $N A$ not analysed due to no abnormal values in analysis. NfL was logtransformed to achieve a less skewed distribution 
SLE and pSS patients, and also that NfL concentrations were higher in patients with pSS compared with SLE patients (Table 2). However, the pSS patients were older than the SLE patients, and when adjusted for age, there were no differences in NfL concentrations between the diseases. This indicates that the neuronal pathogenetic impact is more or less similar in the two diseases.

A number of other biomarkers in CSF and blood were studied. Of these, anti-P antibodies have been shown to exert a pathogenetic effect on the brain in several studies, being associated with psychosis and depression in SLE patients [18]. We found no convincing contribution of intrathecal anti-P antibodies to NfL elevations, possibly indicating that they do not exert a general neuronal destructive effect. On the other hand, none of our patients had psychosis and anti-P levels were generally low and possibly not of a pathogenetic type.

S100B is produced by activated astrocytes as response to damage, danger or other homeostatic disturbances and signal through RAGE and TLR-4 on microglia thus initiating proinflammatory cytokine production and increasing intrathecal immune activity $[31,32]$. Increasing NfL levels were associated with increasing S100B levels in pSS, but not the SLE patients. This observation could point to some different mechanism for brain involvement in the two diseases, but remains speculative. The association disappeared in multivariable testing, again showing that anti-NR2 antibodies were the dominating pathogenetic actors.

Q-albumin-an indirect measure of blood-brain barrier integrity-is calculated by the ratio of CSF versus blood albumin [17]. Increased Q-albumin is seen when the blood-brain barrier is leaking, and albumin passes from blood into the CSF, followed by increased passage of other proteins, including antibodies, cytokines and other bioactive molecules. Disrupted integrity of the blood-brain barrier has lately been advocated as an important and necessary step for neuropsychiatric SLE to develop [33], although some have questioned this [19, 34]. In this study, there was a tendency towards increasing Q-albumin increased NfL levels in the SLE patients, but not pSS. In multivariate statistics, no effect of Q-albumin was seen. Anti-NR2 antibodies remained as the only operative factor for NfL concentrations indicating that at least for pathophysiological processes reflected in increased NfL, the blood-brain barrier is of less importance.

Eight patients, one with SLE and seven with pSS had elevated $\operatorname{IgG}$ indices, reflecting intrathecal IgG synthesis. However, no associations between $\operatorname{IgG}$ indices and NfL levels were observed.

TWEAK is a member of the TNF superfamily of cytokines and acts through the receptor Fn14. Both are present in the CNS on endothelial cells, perivascular astrocytes, neurons and microglia [35, 36]. The function is unclear, complex, and probably depends on the local pathophysiological conditions. Some animal studies indicate that TWEAK opens up the blood-brain barrier, but this has not been confirmed in human studies $[19,37]$. We have hypothesized that TWEAK is produced in human SLE and pSS as response to immunological stress and works as a "neuroprotective" protein in the CNS [19]. In the present study, increased levels of TWEAK was only weakly associated with increasing NfL levels in pSS patients, and this association disappeared in multivariable statistics. This fits the hypothesis that TWEAK does not facilitate the action of brain reactive antibodies by opening the BBB, but could rather be engaged in cellular homeostasis during inflammation and cellular stress.

APL antibodies were measured in blood and there were higher concentrations in the SLE than the pSS patients, as expected. There were more cerebral infarcts in the SLE group, but aPL did not influence NfL levels, indicating that their presence did not exert a chronic pathogenetic effect on the cerebral neurons. This finding is in line with the understanding that cognitive dysfunction in patients with aPL antibodies is caused by cerebral infarcts, and not due to an anti-neuronal effect of the antibodies.

A limitation of the study is the cross-sectional design and lack of a control group and a longitudinal design. Furthermore, there was a relatively low number of participants reflecting that both diseases are relatively rare diseases. In addition, it is hard to obtain high numbers of CSF samples for research in general, and from patients with rare diseases in particular. Relatively low disease activity in the SLE patients could possibly have influenced the results. On the other side, most neuropsychiatric manifestations are immunological targeted to specific structures, and not dependent on high inflammatory activity. The applied methods for cerebral image analyses were probably not sensitive enough to pick up minor morphological changes that we and others previously have found to be associated with the presence of anti-NR2 antibodies. No explicit adjustment for multiple testing was done, and we thus acknowledge that some small $p$ values might have been obtained by chance. In particular, $p$ values close to 0.05 should be interpreted with caution.

Strengths of the study are unselected patients with welldefined diseases, comprehensive and systematic clinical examination, and sampling of CSF and blood under standard conditions.

We conclude that increased concentrations of $\mathrm{NfL}$ in CSF is a marker of brain involvement in patients with pSS as well as in SLE and is reflected in cognitive impairment in several domains. Anti-NR2 antibodies are probably the pathogenetic actors that lead to neuronal distortions. This study is exploratory, and the observations need to be replicated in a prospective, hypothesis-testing design. 
Author contributions ABT, MD PhD: Stavanger University Hospital, Stavanger. Designed and conceptualized study; analyzed the data; drafted the manuscript for intellectual content. MBL, MD PhD: Stavanger University Hospital, Stavanger. Contributed to MRI- and statistical analyses and interpretation; revised the manuscript for intellectual content. HZ MD, PhD: Sahlgrenska University Hospital, Mölndal. Performed NfL analyses; interpreted the data; revised the manuscript for intellectual content. JTK, PhD: University in Stavanger, Stavanger. Statistical analyses; revised the manuscript for intellectual content. IK, MSc: Stavanger University Hospital, Stavanger. Contributed to acquisition of data and sample analyse; revised the manuscript for intellectual content. SMM, PsyD: Stavanger University Hospital, Stavanger. Role in the acquisition of neuropsychological data; Responsible for the design and implementation of the neuropsychological data; clinical interpretation of the data; revised the manuscript for intellectual content. OJG, MD: Stavanger University Hospital, Stavanger. MRI interpretation; revised the manuscript for intellectual content. MB, MD, PhD: Oslo University Hospital, Oslo. MRI interpretation; revised the manuscript for intellectual content. SH, MD, PhD: Kitasato University School of medicine, Kanagawa. Role in the acquisition of data; performed anti-P assays; revised the manuscript for intellectual content. CP, MD: Albert Einstein College of Medicine and Montefiore Medical Center, NY. Performed TWEAK analyses; interpreted the data; revised the manuscript for intellectual content, GA, MD, PhD: Stavanger University Hospital, Stavanger. Interpreted the data; revised the manuscript for intellectual content, Erna Harboe, MD, PhD: Stavanger University Hospital, Stavanger. Major role in acquisition of data; revised the manuscript for intellectual content. KB, MD, PhD: Sahlgrenska University Hospital, Mölndal. Performed NfL analyses; interpreted the data; revised the manuscript for intellectual content. LGG, MD, PhD: Stavanger University Hospital, Stavanger. Major role in acquisition of data; revised the manuscript for intellectual content. RO MD, PhD: Stavanger University Hospital, Stavanger. Designed and conceptualized study; analyzed the data; drafted the manuscript for intellectual content.

Funding Open Access funding provided by University of Bergen. Dr Anne B. Tjensvoll is supported by a grant from the Norwegian Rheumatism Association (Norsk Revmatikerforbund). Dr. Henrik Zetterberg is a Wallenberg Scholar supported by grants from the Swedish Research Council (\#2018-02532), the European Research Council (\#681712), Swedish State Support for Clinical Research (\#ALFGBG-720931), the Alzheimer Drug Discovery Foundation (ADDF), USA (\#2018092016862), and the UK Dementia Research Institute at UCL. Mrs. Ingeborg Kvivik is supported by grant no 912129 from the Western Norway Regional Health Authority (WNRHA). Dr. Shunsei Hirohata is supported by a grant from the Japan Agency for Medical Research and Development (No.15eK0410022h0001). Dr. Guido Alves is supported by grants from the Norwegian Research Council (\#287842), the Norwegian Parkinson Research Foundation, and Reberg's Legacy. Dr. Kaj Blennow is supported by the Swedish Research Council (\#2017-00915), the Alzheimer Drug Discovery Foundation (ADDF), USA (\#RDAPB-201809-2016615), the Swedish Alzheimer Foundation (\#AF-742881), Hjärnfonden, Sweden (\#FO2017-0243), the Swedish state, under the agreement between the Swedish government and the County Councils, the ALF-agreement (\#ALFGBG-715986), and the European Union Joint Program for Neurodegenerative Disorders (JPND2019-466-236). Dr. Roald Omdal. The Western Norway Regional Health Authority (WNRHA) funded the study (grant No.911807).

Data availability The data that support the findings of this study are available from the corresponding author upon reasonable request.

\section{Compliance with ethical standards}

Conflicts of interest Dr. Henrik Zetterberg reports that he served on scientific advisory boards for Denali, Roche Diagnostics, Wave, Samumed, and $\operatorname{CogRx}$, has given lectures in symposia sponsored by $\mathrm{Fu}$ jirebio, Alzecure, and Biogen, and is a co-founder of Brain Biomarker Solutions in Gothenburg AB, a GU Ventures-based platform company at the University of Gothenburg. Dr. Mona Beyer has received honoraria for lecturing, from Novartis and Biogen Idec, Merck AB, Roche Norge, and Sanofi Genzyme. Dr. Kaj Blennow has served as a consultant or on advisory boards for Abcam, Axon, Biogen, Lilly, MagQu, Novartis, and Roche Diagnostics, and is a co-founder of Brain Biomarker Solutions in Gothenburg AB (BBS), which is a part of the GU Ventures Incubator Program. Dr. Roald Omdal has received speaker honoraria from several pharmaceutical companies. The other authors report no conflicts of interest.

Ethics approval This study complied with the Helsinki Declaration and was approved by the Regional Research Ethics Committee (REKvest 2010/1455).

Consent to participate Written informed consent was obtained from all patients.

\section{Consent for publication NA.}

Open Access This article is licensed under a Creative Commons Attribution 4.0 International License, which permits use, sharing, adaptation, distribution and reproduction in any medium or format, as long as you give appropriate credit to the original author(s) and the source, provide a link to the Creative Commons licence, and indicate if changes were made. The images or other third party material in this article are included in the article's Creative Commons licence, unless indicated otherwise in a credit line to the material. If material is not included in the article's Creative Commons licence and your intended use is not permitted by statutory regulation or exceeds the permitted use, you will need to obtain permission directly from the copyright holder. To view a copy of this licence, visit http://creativecommons.org/licenses/by/4.0/.

\section{References}

1. Schwartz N, Stock AD, Putterman C (2019) Neuropsychiatric lupus: new mechanistic insights and future treatment directions. Nat Rev Rheumatol 15(3):137-152. https://doi.org/10.1038/s4158 4-018-0156-8

2. Mariette X, Criswell LA (2018) Primary Sjogren's syndrome. N Engl J Med 378(10):931-939. https://doi.org/10.1056/NEJMc p1702514

3. Harboe E, Tjensvoll AB, Maroni S, Goransson LG, Greve OJ, Beyer MK, Herigstad A, Kvaloy JT, Omdal R (2009) Neuropsychiatric syndromes in patients with systemic lupus erythematosus and primary Sjogren syndrome: a comparative populationbased study. Ann Rheum Dis 68(10):1541-1546. https://doi. org/10.1136/ard.2008.098301

4. Gaetani L, Blennow K, Calabresi P, Di Filippo M, Parnetti L, Zetterberg H (2019) Neurofilament light chain as a biomarker in neurological disorders. J Neurol Neurosurg Psychiatry 90(8):870 881. https://doi.org/10.1136/jnnp-2018-320106

5. Hanly JG, Hong C, Smith S, Fisk JD (1999) A prospective analysis of cognitive function and anticardiolipin antibodies in systemic 
lupus erythematosus. Arthritis Rheum 42(4):728-734. https:// doi.org/10.1002/1529-0131(199904)42:4\%3c728::AID-ANR16 \%3e3.0.CO;2-O

6. Magro-Checa C, Kumar S, Ramiro S, Beaart-van de Voorde LJ, Eikenboom J, Ronen I, de Bresser J, van Buchem MA, Huizinga TW, Steup-Beekman GM (2019) Are serum autoantibodies associated with brain changes in systemic lupus erythematosus? MRI data from the Leiden NP-SLE cohort. Lupus 28(1):94-103. https ://doi.org/10.1177/0961203318816819

7. Omdal R, Brokstad K, Waterloo K, Koldingsnes W, Jonsson R, Mellgren SI (2005) Neuropsychiatric disturbances in SLE are associated with antibodies against NMDA receptors. Eur J Neurol 12(5):392-398. https://doi.org/10.1111/j.1468-1331.2004.00976 .X

8. Lauvsnes MB, Maroni SS, Appenzeller S, Beyer MK, Greve OJ, Kvaloy JT, Harboe E, Goransson LG, Tjensvoll AB, Omdal R (2013) Memory dysfunction in primary Sjogren's syndrome is associated with anti-NR2 antibodies. Arthritis Rheum 65(12):3209-3217. https://doi.org/10.1002/art.38127

9. Viana VT, Durcan L, Bonfa E, Elkon KB (2017) Ribosomal P antibody: 30 years on the road. Lupus 26(5):453-462. https://doi. org/10.1177/0961203317690243

10. Hochberg MC (1997) Updating the American College of Rheumatology revised criteria for the classification of systemic lupus erythematosus. Arthritis Rheum 40(9):1725. https://doi. org/10.1002/1529-0131(199709)40:9\%3c1725::AID-ART29 $\% 3 \mathrm{e} 3.0 . \mathrm{CO} ; 2-\mathrm{Y}$

11. Vitali C, Bombardieri S, Jonsson R, Moutsopoulos HM, Alexander EL, Carsons SE, Daniels TE, Fox PC, Fox RI, Kassan SS, Pillemer SR, Talal N, Weisman MH (2002) Classification criteria for Sjogren's syndrome: a revised version of the European criteria proposed by the American-European Consensus Group. Ann Rheum Dis 61(6):554-558

12. Bombardier C, Gladman DD, Urowitz MB, Caron D, Chang CH (1992) Derivation of the SLEDAI. A disease activity index for lupus patients. The committee on prognosis studies in SLE. Arthritis Rheum 35 (6):630-640

13. Gladman D, Ginzler E, Goldsmith C, Fortin P, Liang M, Urowitz M, Bacon P, Bombardieri S, Hanly J, Hay E, Isenberg D, Jones J, Kalunian K, Maddison P, Nived O, Petri M, Richter M, SanchezGuerrero J, Snaith M, Sturfelt G, Symmons D, Zoma A (1996) The development and initial validation of the systemic lupus international collaborating Clinics/American college of rheumatology damage index for systemic lupus erythematosus. Arthritis Rheum 39(3):363-369

14. The International Classification of Headache Disorders: 2nd edition (2004). Cephalalgia 24(Suppl 1_:9-160. doi:10.1111/j.1468-2982.2003.00824.x

15. Beck AT, Beamesderfer A (1974) Assessment of depression: the depression inventory. Mod Probl Pharmacopsychiatry 7:151-169

16. Wolfe F (2004) Fatigue assessments in rheumatoid arthritis: comparative performance of visual analog scales and longer fatigue questionnaires in 7760 patients. J Rheumatol 31(10):1896-1902

17. Tibbling G, Link H, Ohman S (1977) Principles of albumin and $\mathrm{IgG}$ analyses in neurological disorders. I. Establishment of reference values. Scandinavian J Clin Laboratory Investigation 37(5):385-390. https://doi.org/10.1080/00365517709091496

18. Hirohata S, Arinuma Y, Takayama M, Yoshio T (2007) Association of cerebrospinal fluid anti-ribosomal $p$ protein antibodies with diffuse psychiatric/neuropsychological syndromes in systemic lupus erythematosus. Arthritis Res Ther 9(3):R44. https://doi. org/10.1186/ar2184

19. Lauvsnes MB, Tjensvoll AB, Maroni SS, Kvivik I, Grimstad T, Greve OJ, Harboe E, Goransson LG, Putterman C, Omdal R (2018) The blood-brain barrier, TWEAK, and neuropsychiatric involvement in human systemic lupus erythematosus and primary Sjogren's syndrome. Lupus 27(13):2101-2111. https:// doi.org/10.1177/0961203318804895

20. Scheltens P, Barkhof F, Leys D, Pruvo JP, Nauta JJ, Vermersch P, Steinling M, Valk J (1993) A semiquantative rating scale for the assessment of signal hyperintensities on magnetic resonance imaging. J Neurol Sci 114(1):7-12

21. Lauvsnes MB, Beyer MK, Kvaloy JT, Greve OJ, Appenzeller S, Kvivik I, Harboe E, Tjensvoll AB, Goransson LG, Omdal R (2014) Association of hippocampal atrophy with cerebrospinal fluid antibodies against the NR2 subtype of the N-methyl-D-aspartate receptor in patients with systemic lupus erythematosus and patients with primary sjogren's syndrome. Arthritis \& rheumatology (Hoboken, NJ) 66(12):3387-3394. https://doi.org/10.1002/ art.38852

22. Wechsler D (1987) WMS-R: Wechsler Memory Scale-Revised : Manual. Harcourt Brace Jovanovich

23. Wechsler D (1955) Manual for the Wechsler Adult Intelligence Scale. Manual for the Wechsler Adult Intelligence Scale. Psychological Corp., Oxford, England

24. Stroop JR (1935) Studies of interference in serial verbal reactions. J Exp Psychol 18:643-662

25. Heaton RK, Chelune GJ, Talley JL, Kay GG, Curtiss G (1993) Wisconsin card sorting test manual: revised and expanded. Psychological Assessment Resources Inc, Odessa

26. Benton AL, Hamsher K (1977) Multilingual aphasia examination. University of Iowa

27. Reitan RM, Wolfson D (1985) The Halstead-Reitan neuropsychological test battery: theory and clinical interpretation. Neuropsychology Press

28. Faust TW, Chang EH, Kowal C, Berlin R, Gazaryan IG, Bertini E, Zhang J, Sanchez-Guerrero J, Fragoso-Loyo HE, Volpe BT, Diamond B, Huerta PT (2010) Neurotoxic lupus autoantibodies alter brain function through two distinct mechanisms. Proc Natl Acad Sci USA 107(43):18569-18574. https://doi.org/10.1073/ pnas. 1006980107

29. Khalil M, Teunissen CE, Otto M, Piehl F, Sormani MP, Gattringer T, Barro C, Kappos L, Comabella M, Fazekas F, Petzold A, Blennow K, Zetterberg H, Kuhle J (2018) Neurofilaments as biomarkers in neurological disorders. Nat Rev Neurol 14(10):577-589. https://doi.org/10.1038/s41582-018-0058-z

30. Trysberg E, Nylen K, Rosengren LE, Tarkowski A (2003) Neuronal and astrocytic damage in systemic lupus erythematosus patients with central nervous system involvement. Arthritis Rheum 48(10):2881-2887. https://doi.org/10.1002/art.11279

31. Hofmann MA, Drury S, Fu C, Qu W, Taguchi A, Lu Y, Avila C, Kambham N, Bierhaus A, Nawroth P, Neurath MF, Slattery T, Beach D, McClary J, Nagashima M, Morser J, Stern D, Schmidt AM (1999) RAGE mediates a novel proinflammatory axis: a central cell surface receptor for S100/calgranulin polypeptides. Cell 97(7):889-901. https://doi.org/10.1016/s0092-8674(00)80801-6

32. Bertheloot D, Latz E (2017) HMGB1, IL-1alpha, IL-33 and S100 proteins: dual-function alarmins. Cell Mol Immunol 14(1):43-64. https://doi.org/10.1038/cmi.2016.34

33. Chi JM, Mackay M, Hoang A, Cheng K, Aranow C, Ivanidze J, Volpe B, Diamond B, Sanelli PC (2019) Alterations in bloodbrain barrier permeability in patients with systemic lupus erythematosus. AJNR Am J Neuroradiol 40(3):470-477. https://doi. org/10.3174/ajnr.A5990

34. Gulati G, Iffland PH 2nd, Janigro D, Zhang B, Luggen ME (2016) Anti-NR2 antibodies, blood-brain barrier, and cognitive dysfunction. Clin Rheumatol 35(12):2989-2997. https://doi.org/10.1007/ s10067-016-3339-1

35. Polavarapu R, Gongora MC, Winkles JA, Yepes M (2005) Tumor necrosis factor-like weak inducer of apoptosis increases the permeability of the neurovascular unit through nuclear factor-kappa 
B pathway activation. J Neurosci 25(44):10094-10100. https:// doi.org/10.1523/jneurosci.3382-05.2005

36. Yepes M (2007) Tweak and FN14 in central nervous system health and disease. Front Biosci 12:2772-2781
37. Fragoso-Loyo H, Atisha-Fregoso Y, Nunez-Alvarez CA, Llorente L (2016) Utility of TWEAK to assess neuropsychiatric disease activity in systemic lupus erhytematosus. Lupus 25(4):364-369. https://doi.org/10.1177/0961203315610206

\section{Affiliations}

Anne B. Tjensvoll ${ }^{1}$. Maria B. Lauvsnes ${ }^{2} \cdot$ Henrik Zetterberg ${ }^{3,4,5,6} \cdot$ Jan T. Kvaløy ${ }^{7,8} \cdot$ Ingeborg Kvivik $^{7}$. Stian S. Maroni ${ }^{9}$. Ole J. Greve ${ }^{10}$. Mona K. Beyer ${ }^{11,12}$. Shunsei Hirohata ${ }^{13}$. Chaim Putterman ${ }^{14,15,16}$. Guido Alves ${ }^{17,18}$. Erna Harboe $^{2} \cdot$ Kaj Blennow $^{3,4} \cdot$ Lasse G. Gøransson ${ }^{2,19} \cdot$ Roald Omdal $^{2,19}$

1 Department of Neurology, Stavanger University Hospital, Stavanger, Norway

2 Department of Internal Medicine, Clinical Immunology Unit, Stavanger University Hospital, POB 8100, 4068 Stavanger, Norway

3 Clinical Neurochemistry Laboratory, Sahlgrenska University Hospital, Mölndal, Sweden

4 Department of Psychiatry and Neurochemistry, Institute of Neuroscience and Physiology, The Sahlgrenska Academy At the University of Gothenburg, Mölndal, Sweden

5 UK Dementia Research Institute At UCL, London, UK

6 Department of Neurodegenerative Disease, UCL Institute of Neurology, London, UK

7 Research Department, Stavanger University Hospital, Stavanger, Norway

8 Department of Mathematics and Physics, University of Stavanger, Stavanger, Norway

9 Clinical Neuropsychology Unit, Division of Psychiatry, Stavanger University Hospital, Stavanger, Norway

10 Department of Radiology, Stavanger University Hospital, Stavanger, Norway
11 Institute of Clinical Medicine, University of Oslo, Oslo, Norway

12 Division of Radiology and Nuclear Medicine, Oslo University Hospital, Oslo, Norway

13 Department of Rheumatology and Infectious Diseases, Kitasato University School of Medicine, 1-15-1 Kitasato, Sagamihara, Kanagawa 252-0374, Japan

14 Division of Rheumatology, Albert Einstein College of Medicine and Montefiore Medical Center, Bronx, NY, USA

15 Azrieli School of Medicine Bar-Ilan University, Zefat, Israel

16 Galilee Medical Center Research Institute, Nahariya, Israel

17 The Norwegian Centre for Movement Disorders and Department of Neurology, Stavanger University Hospital, Stavanger, Norway

18 Department of Chemistry, Bioscience and Environmental Engineering, University of Stavanger, Stavanger, Norway

19 Department of Clinical Science, Faculty of Medicine, University of Bergen, Bergen, Norway 